\title{
Diabetes mellitus, dyslipoproteinaemias and atherosclerosis
}

\section{G.Steiner}

Department of Medicine at the University of Toronto and the Toronto Hospital, and the World Health Organization Collaborating Centre for the Study of Atherosclerosis in Diabetes, Toronto, Canada

The most common complication of diabetes mellitus is atherosclerosis. Whereas approximately one-third of the North American population will die from atherosclerotic cardiovascular disease, closer to threequarters of those with diabetes will do so. Furthermore, morbidity from coronary artery disease, peripheral vascular disease and strokes is much greater in those with diabetes than it is in those without. This is seen both in populations in which atherosclerotic cardiovascular disease is common and in those in which it occurs infrequently.

Dyslipoproteinaemias are among the many factors that can contribute to this increase in the risk for atherosclerosis. Those with diabetes may have both quantitative and qualitative changes in their lipoproteins. In those whose diabetes is treated without insulin, the major quantitative change is an elevation in the concentration of the triglyceride-rich lipoproteins. Insulin injections, on the other hand, may cause a decline in plasma triglyceride levels. The increase in plasma triglyceride is due mainly to an increase in the number of smaller triglyceride-rich lipoprotein particles. Previously we showed that the number of these particles is increased in those without diabetes who have coronary artery disease. Recently we found that the number of triglyceride-rich lipoprotein particles in those with diabetes who undergo angiography is related to the severity of their coronary artery disease. These effects of the triglyceride-rich lipoproteins are independent of high density lipoprotein (HDL) levels. In diabetes, HDL levels may be lower than, higher than or not different from those in the

Corresponding author: Dr. G. Steiner, Room NUW9-112, The Toronto Hospital (General Division), 200 Elizabeth Street, Toronto, Ontario, Canada, M5G 2C4

A bbreviations: HDL, High density lipoproteins; LDL, low density lipoproteins. general population. They often reflect the nature of the hypoglycaemic regimen, the coexistence of obesity, or the presence of hypertriglyceridaemia. In diabetes the levels of low density lipoprotein (LDL) are similar to those seen in the general population. However, this does not imply that LDL is not a risk factor in those with diabetes. It is. The curve describing coronary artery disease mortality as compared to cholesterol level is similar in shape in those with diabetes to the curve in those without diabetes. However, in those with diabetes the coronary artery disease mortality at any given level of cholesterol is approximately four times greater than it is in those without. This may reflect differences in the quality of the LDL that make any given amount more atherogenic. Such qualitative changes include non-enzymatic glycation of LDL, oxidation of LDL, and/or the presence in the plasma of smaller and denser LDL. LDL glycation reflects the level of glycaemia in the plasma. Some suggest that LDL size and density may reflect the plasma triglyceride concentration only. Others feel that hypertriglyceridaemia does not explain the size and density differences entirely. In addition to the risk effects of fasting hypertriglyceridaemia, postprandial levels of triglyceride, retinyl palmitate, and apoB48 in small triglyceride-rich lipoproteins have been found to be increased in those with coronary artery disease.

Most people with non-insulin-dependent diabetes are resistant to insulin. In order to compensate for this, they may develop chronic hyperinsulinaemia. The production of triglyceride-rich lipoproteins in vivo is increased in the chronically hyperinsulinaemic state. This contrasts with the effects of acute insulin administration, in which triglyceride-rich lipoprotein production may decrease. These changes may reflect the balance of a number of processes ranging from the supply of substrate non-esterified fatty acids to alterations in the many intrahepatic processes involved 
in the production of very low density lipoproteins. The overall plasma triglyceride levels are determined by the rate of production and the rate of removal of the triglyceride-rich lipoproteins. The latter are limited by lipoprotein lipase, an enzyme the activity of which is increased by insulin. The product of triglyceride-rich lipoprotein degradation is a smaller remnant, a particle that has been shown to be atherogenic. Thus, an increase in the turnover of the triglyceride-rich lipoproteins in diabetes could generate lipoprotein particles that increase the risk of atherosclerosis.
At this time, guidelines with respect to the treatment of the dyslipoproteinaemias of diabetes are based on extrapolation from studies of non-diabetic populations, and on post-hoc analyses of subgroups with diabetes that have been included in larger lipid intervention trials. Currently, one study is being conducted - the Diabetes Atherosclerosis Intervention Study (DAIS) - that has been primarily designed to determine whether treating the dyslipoproteinemia of diabetes will reduce the risk of coronary artery disease in non-insulin-dependent diabetes. At present it is in its active treatment phase. 